\title{
A New Theorem on Product Summability of Infinite Series
}

\author{
ARADHANA DUTT JAUHARI \\ Noida Institute Of Eng. \& Technology, Gr. Noida,U.P. (India)
}

\begin{abstract}
A given theorem is a some advanced proof in product summability of infinite series.Many other results some known and unknown are derived.
\end{abstract}

Key Words And Phrases: summability, absolute summability, product summability.

\section{Introduction :}

Let $\sum \mathrm{a}_{\mathrm{n}}$ be a given infinite series with partial sum $\mathrm{S}_{\mathrm{n}}$. Let $\mathrm{u}_{\mathrm{n}}^{\alpha}$ denote the $\mathrm{n}^{\text {th }}$ Cesàro mean of order $\alpha>-1$ of the sequence $\left\{s_{n}\right\}$.

The series $\sum \mathrm{a}_{\mathrm{n}}$ is said to be summable $|\mathrm{C}, \alpha|_{\mathrm{k}}, \mathrm{k} \geq 1$, if

$$
\sum_{n=1}^{\infty} n^{k-1}\left|u_{n}^{\alpha}-u_{n-1}^{\alpha}\right|^{k}<\infty
$$

Let $\left\{p_{n}\right\}$ be a sequence of positive real constants, such that

$$
\mathrm{P}_{\mathrm{n}}=\mathrm{p}_{0}+\mathrm{p}_{1}+\ldots \ldots \mathrm{p}_{\mathrm{n}} \quad \text { as }, \mathrm{n} \rightarrow \infty \quad\left(\mathrm{P}_{-1}=\mathrm{p}_{-1}=0\right)
$$

The $(\mathrm{N}, \mathrm{p})$ transform of $\phi_{\mathrm{n}}$ of $\left\{\mathrm{s}_{\mathrm{n}}\right\}$ generated by $\left\{\mathrm{p}_{\mathrm{n}}\right\}$ is defined by -

$$
\phi_{\mathrm{n}}=\frac{1}{\mathrm{P}_{\mathrm{n}}} \sum_{\mathrm{v}=0}^{\mathrm{m}} \mathrm{p}_{\mathrm{n}-\mathrm{v}} \mathrm{s}_{\mathrm{v}}
$$

The sequence-to-sequence transformation-

$$
\phi_{\mathrm{n}}=\frac{1}{\mathrm{P}_{\mathrm{n}}} \sum_{\mathrm{v}=0}^{\mathrm{n}} \mathrm{p}_{\mathrm{v}} \mathrm{s}_{\mathrm{v}}
$$

defines the sequence $\left\{\phi_{n}\right\}$ of $\left(\bar{N}, p_{n}\right)$ transform of $\left\{s_{n}\right\}$ generated by $\left\{p_{n}\right\}$, The series $\sum a_{n}$ is said to be summable $\left|R, p_{n}\right|_{k}, k \geq 1$, if -

$$
\sum_{n=1}^{\infty} n^{k-1}\left|\phi_{n}-\phi_{n-1}\right|^{k}<\infty
$$

In special case when $\mathrm{p}_{\mathrm{n}}=1, \forall \mathrm{n}\left|\mathrm{R}, \mathrm{p}_{\mathrm{n}}\right|_{\mathrm{k}}$ summability reduces to $|\mathrm{C}, 1|_{\mathrm{k}}$ summability.

The series $\sum \mathrm{a}_{\mathrm{n}}$ is said to be summable $|(\overline{\mathrm{N}}, \mathrm{p})(\mathrm{N}, \mathrm{q})|$ when the $(\mathrm{N}, \mathrm{p})$ transform of the $(\mathrm{N}, \mathrm{q})$ transform of $\left\{\mathrm{s}_{\mathrm{n}}\right\}$ is a sequence of bounded variation.

Let $\left\{T_{n}\right\}$ defines the sequence of $\left(\bar{N}, q_{n}\right)$ transform of the $\left(\bar{N}, p_{n}\right)$ transform of $\left\{s_{n}\right\}$ generated by the sequence $\left\{q_{n}\right\}$ and $\left\{p_{n}\right\}$ respectively .

The series $\sum \mathrm{a}_{\mathrm{n}}$ is said to be summable $\left|\left(\mathrm{R}, \mathrm{q}_{\mathrm{n}}\right)\left(\mathrm{R}, \mathrm{p}_{\mathrm{n}}\right)\right|_{\mathrm{k}}, \mathrm{k} \geq 1$, if 


$$
\mathcal{A}_{k}=\left\{\left(\mathrm{T}_{\mathrm{n}}\right) ; \sum_{\mathrm{n}=1}^{\infty} \mathrm{n}^{\mathrm{k}-1}\left|\mathrm{a}_{\mathrm{n}}\right|^{\mathrm{k}}<\infty ; \mathrm{a}_{\mathrm{n}}=\mathrm{T}_{\mathrm{n}}-\mathrm{T}_{\mathrm{n}-1}\right\}
$$

and

$$
\mathcal{A}_{k}^{\delta}=\left\{\left(\mathrm{T}_{\mathrm{n}}\right) ; \sum_{\mathrm{n}=1}^{\infty} \mathrm{n}^{\delta \mathrm{k}+\mathrm{k}-1}\left|\mathrm{a}_{\mathrm{n}}\right|^{\mathrm{k}}<\infty ; \mathrm{a}_{\mathrm{n}}=\mathrm{T}_{\mathrm{n}}-\mathrm{T}_{\mathrm{n}-1}\right\}
$$

We may assume ,

$$
\begin{aligned}
& \mathrm{Q}_{\mathrm{n}}=\mathrm{q}_{0}+\mathrm{q}_{1}+\mathrm{q}_{2}+\ldots \ldots \mathrm{q}_{\mathrm{n}} ; \mathrm{n} \rightarrow \infty \\
& \mathrm{R}_{\mathrm{n}}=\mathrm{r}_{0}+\mathrm{r}_{1}+\mathrm{r}_{2}+\ldots \ldots . \mathrm{r}_{\mathrm{n}} ; \mathrm{n} \rightarrow \infty
\end{aligned}
$$

In 2008, SULAIMAN [3] proved the theorem. The main objective of this paper is to generalize the theorem of SULAIMAN[3]. However our theorem is as follows.

\section{Theorem 1}

Let $\mathrm{k} \geq 1$ and $\delta \geq 0,\left\{\lambda_{\mathrm{n}}\right\}$ be a sequence of constants,

$$
\mathrm{f}_{\mathrm{v}}=\sum_{\mathrm{r}=\mathrm{v}}^{\mathrm{n}} \frac{\mathrm{q}_{\mathrm{r}}}{\mathrm{P}_{\mathrm{r}}}, \quad \mathrm{F}_{\mathrm{v}}=\sum_{\mathrm{r}=\mathrm{v}}^{\mathrm{n}} \mathrm{p}_{\mathrm{r}} \mathrm{f}_{\mathrm{r}}
$$

Let

$$
\begin{aligned}
& \mathrm{p}_{\mathrm{n}} \mathrm{Q}_{\mathrm{n}}=\square\left(\mathrm{P}_{\mathrm{n}}\right) \\
& \sum_{\mathrm{n}=\mathrm{v}+1}^{\infty} \frac{\mathrm{n}^{\delta \mathrm{k}+\mathrm{k}-1} \mathrm{q}_{\mathrm{n}}^{\mathrm{k}}}{\mathrm{Q}_{\mathrm{n}}^{\mathrm{k}} \mathrm{Q}_{\mathrm{n}-1}}=\square\left\{\frac{\mathrm{v}^{\delta \mathrm{k}+\mathrm{k}-1} \mathrm{q}_{\mathrm{v}}^{\mathrm{k}-1}}{\mathrm{Q}_{\mathrm{v}}^{\mathrm{k}}}\right\}
\end{aligned}
$$

Then, sufficient conditions for implications-

$\sum \mathrm{a}_{\mathrm{n}}$ is summable $\left|\mathrm{R}, \mathrm{r}_{\mathrm{n}}, \delta\right|_{\mathrm{k}} \Rightarrow \sum \mathrm{a}_{\mathrm{n}} \lambda_{\mathrm{n}}$ is summable

$$
\left|\left(\mathrm{R}, \mathrm{q}_{\mathrm{n}}\right)\left(\mathrm{R}, \mathrm{p}_{\mathrm{n}}\right), \delta\right|_{\mathrm{k}}
$$

are,

$$
\begin{aligned}
& \left|\lambda_{\mathrm{v}}\right| \mathrm{F}_{\mathrm{v}}=\square\left(\mathrm{Q}_{\mathrm{v}}\right) \\
& \left|\lambda_{\mathrm{n}}\right|=\square\left(\mathrm{Q}_{\mathrm{n}}\right) \\
& \mathrm{p}_{\mathrm{v}} \mathrm{R}_{\mathrm{v}}\left|\lambda_{\mathrm{v}}\right|=\square\left(\mathrm{Q}_{\mathrm{v}}\right) \\
& \mathrm{p}_{\mathrm{v}} \mathrm{q}_{\mathrm{v}} \mathrm{R}_{\mathrm{v}}\left|\lambda_{\mathrm{v}}\right|=\square\left(\mathrm{Q}_{\mathrm{v}} \mathrm{Q}_{\mathrm{v}-1} \mathrm{r}_{\mathrm{v}}\right) \\
& \mathrm{p}_{\mathrm{v}} \mathrm{q}_{\mathrm{v}} \mathrm{R}_{\mathrm{n}}\left|\lambda_{\mathrm{n}}\right|=\square\left(\mathrm{P}_{\mathrm{n}} \mathrm{Q}_{\mathrm{n}} \mathrm{r}_{\mathrm{n}}\right) \\
& \mathrm{R}_{\mathrm{v}-1}\left|\Delta \lambda_{\mathrm{v}}\right| \mathrm{F}_{\mathrm{v}+1}=\square\left(\mathrm{Q}_{\mathrm{v}} \mathrm{r}_{\mathrm{v}}\right) \\
& \mathrm{R}_{\mathrm{v}-1}\left|\Delta \lambda_{\mathrm{v}}\right|=\square\left(\mathrm{Q}_{\mathrm{v}} \mathrm{r}_{\mathrm{v}}\right)
\end{aligned}
$$

\section{THEOREM 2}

Let ( 2.3) be satisfied and

$$
\begin{aligned}
& \mathrm{P}_{\mathrm{v}}=\square\left(\mathrm{p}_{\mathrm{v}} \mathrm{Q}_{\mathrm{v}}\right) \\
& \mathrm{Q}_{\mathrm{n}}=\square\left(\mathrm{nq}_{\mathrm{n}}\right)
\end{aligned}
$$

then, necessary conditions for implication (2.4) to be satisfied are, 


$$
\begin{aligned}
& \left|\lambda_{\mathrm{n}}\right|=\square\left(\frac{\mathrm{Q}_{\mathrm{v}} \mathrm{Q}_{\mathrm{v}-1} \mathrm{r}_{\mathrm{v}}}{\left(1+\mathrm{F}_{\mathrm{v}}\right) \mathrm{q}_{\mathrm{v}} \mathrm{R}_{\mathrm{v}}}\right), \\
& \left|\Delta \lambda_{\mathrm{v}}\right|=\square\left(\frac{\mathrm{v}^{(1+\delta)-1 / \mathrm{k}} \mathrm{r}_{\mathrm{v}} \mathrm{Q}_{\mathrm{v}}}{\left(1+\mathrm{F}_{\mathrm{v}+1}\right) \mathrm{R}_{\mathrm{v}}}\right)
\end{aligned}
$$

\section{Proof Of The Theorem 1:}

Let $\left\{S_{n}\right\}$ be the sequence of partial sums of $\sum a_{n} \lambda_{n}$. Let $v_{n}$ and $V_{n}$ be the $\left(\bar{N}, r_{n}\right)$, $\left(\overline{\mathrm{N}}, \mathrm{q}_{\mathrm{n}}\right),\left(\overline{\mathrm{N}}, \mathrm{p}_{\mathrm{n}}\right)$ transforms of the sequences $\left\{\mathrm{s}_{\mathrm{n}}\right\},\left\{\mathrm{S}_{\mathrm{n}}\right\}$ respectively,

We write,

Therefore,

$$
\mathrm{t}_{\mathrm{n}}=\mathrm{v}_{\mathrm{n}}-\mathrm{v}_{\mathrm{n}-1}, \quad \mathrm{~T}_{\mathrm{n}}=\mathrm{V}_{\mathrm{n}}-\mathrm{V}_{\mathrm{n}-1}
$$

$$
\begin{aligned}
& \mathrm{t}_{\mathrm{n}}=\frac{\mathrm{r}_{\mathrm{n}}}{\mathrm{R}_{\mathrm{n}} \mathrm{R}_{\mathrm{n}-1}} \sum_{\mathrm{v}=1}^{\mathrm{n}} \mathrm{R}_{\mathrm{v}-1} \mathrm{a}_{\mathrm{v}} \\
& \mathrm{V}_{\mathrm{n}}=\frac{1}{\mathrm{Q}_{\mathrm{n}}} \sum_{\mathrm{r}=0}^{\mathrm{n}} \mathrm{q}_{\mathrm{r}} \frac{1}{\mathrm{P}_{\mathrm{r}}} \sum_{\mathrm{v}=0}^{\mathrm{r}} \mathrm{p}_{\mathrm{v}} \mathrm{S}_{\mathrm{v}} \\
& =\frac{1}{Q_{n}} \sum_{v=0}^{n} p_{v} S_{v} \sum_{r=v}^{n} \frac{q_{r}}{P_{r}} \\
& =\frac{1}{Q_{n}} \sum_{v=0}^{n} p_{v} S_{v} f_{v} \\
& \text { Also, } \quad \mathrm{T}_{\mathrm{n}}=\mathrm{V}_{\mathrm{n}}-\mathrm{V}_{\mathrm{n}-1} \\
& =\frac{\mathrm{q}_{\mathrm{n}}}{\mathrm{Q}_{\mathrm{n}} \mathrm{Q}_{\mathrm{n}-1}} \sum_{\mathrm{r}=0}^{\mathrm{n}} \mathrm{p}_{\mathrm{r}} \mathrm{S}_{\mathrm{r}} \mathrm{f}_{\mathrm{r}}+\frac{\mathrm{p}_{\mathrm{n}} \mathrm{S}_{\mathrm{n}} \mathrm{f}_{\mathrm{n}}}{\mathrm{Q}_{\mathrm{n}-1}} \\
& =\frac{\mathrm{q}_{\mathrm{n}}}{\mathrm{Q}_{\mathrm{n}} \mathrm{Q}_{\mathrm{n}-1}} \sum_{\mathrm{r}=0}^{\mathrm{v}} \mathrm{p}_{\mathrm{r}} \mathrm{f}_{\mathrm{r}} \sum_{\mathrm{v}=0}^{\mathrm{r}} \mathrm{a}_{\mathrm{v}} \lambda_{\mathrm{v}}+\frac{\mathrm{p}_{\mathrm{n}} \mathrm{q}_{\mathrm{n}}}{\mathrm{P}_{\mathrm{n}} \mathrm{Q}_{\mathrm{n}-1}} \sum_{\mathrm{v}=0}^{\mathrm{n}} \mathrm{a}_{\mathrm{v}} \lambda_{\mathrm{v}} \\
& =\frac{\mathrm{q}_{\mathrm{n}}}{\mathrm{Q}_{\mathrm{n}} \mathrm{Q}_{\mathrm{n}-1}} \sum_{\mathrm{v}=0}^{\mathrm{n}} \mathrm{a}_{\mathrm{v}} \lambda_{\mathrm{v}} \sum_{\mathrm{r}=\mathrm{v}}^{\mathrm{n}} \mathrm{p}_{\mathrm{r}} \mathrm{f}_{\mathrm{r}}+\frac{\mathrm{p}_{\mathrm{n}} \mathrm{q}_{\mathrm{n}}}{\mathrm{P}_{\mathrm{n}} \mathrm{Q}_{\mathrm{n}-1}} \sum_{\mathrm{v}=0}^{\mathrm{n}} \mathrm{a}_{\mathrm{v}} \lambda_{\mathrm{v}} \\
& =\frac{\mathrm{q}_{\mathrm{n}}}{\mathrm{Q}_{\mathrm{n}} \mathrm{Q}_{\mathrm{n}-1}} \sum_{\mathrm{v}=1}^{\mathrm{n}} \mathrm{R}_{\mathrm{v}-1} \mathrm{a}_{\mathrm{v}} \frac{\lambda_{\mathrm{v}}}{\mathrm{R}_{\mathrm{v}-1}} \sum_{\mathrm{r}=\mathrm{v}}^{\mathrm{n}} \mathrm{p}_{\mathrm{r}} \mathrm{f}_{\mathrm{r}}+ \\
& +\frac{\mathrm{p}_{\mathrm{n}} \mathrm{q}_{\mathrm{n}}}{\mathrm{P}_{\mathrm{n}} \mathrm{Q}_{\mathrm{n}-1}} \sum_{\mathrm{v}=1}^{\mathrm{n}} \mathrm{R}_{\mathrm{v}-1} \mathrm{a}_{\mathrm{v}} \frac{\lambda_{\mathrm{v}}}{\mathrm{R}_{\mathrm{v}-1}} \\
& =\frac{\mathrm{q}_{\mathrm{n}}}{\mathrm{Q}_{\mathrm{n}} \mathrm{Q}_{\mathrm{n}-1}} \sum_{\mathrm{v}=1}^{\mathrm{n}-1}\left(\sum_{\mathrm{r}=1}^{\mathrm{v}} \mathrm{R}_{\mathrm{r}-1} \mathrm{a}_{\mathrm{r}}\right) \Delta_{\mathrm{v}}\left(\frac{\lambda_{\mathrm{v}}}{\mathrm{R}_{\mathrm{v}-1}} \sum_{\mathrm{r}=\mathrm{v}}^{\mathrm{n}} \mathrm{p}_{\mathrm{r}} \mathrm{f}_{\mathrm{r}}\right)+\left(\sum_{\mathrm{v}=1}^{\mathrm{n}} \mathrm{R}_{\mathrm{v}-1} \mathrm{a}_{\mathrm{v}}\right) \frac{\mathrm{p}_{\mathrm{n}} \mathrm{f}_{\mathrm{n}} \lambda_{\mathrm{n}}}{\mathrm{R}_{\mathrm{n}-1}}+
\end{aligned}
$$




$$
\begin{aligned}
& \quad+\frac{\mathrm{p}_{\mathrm{n}} \mathrm{q}_{\mathrm{n}}}{\mathrm{P}_{\mathrm{n}} \mathrm{Q}_{\mathrm{n}-1}}\left(\sum_{\mathrm{v}=1}^{\mathrm{n}-1}\left(\sum_{\mathrm{r}=1}^{\mathrm{v}} \mathrm{R}_{\mathrm{r}-1} \mathrm{a}_{\mathrm{r}}\right) \Delta\left(\frac{\lambda_{\mathrm{v}}}{\mathrm{R}_{\mathrm{v}-1}}\right)\right)+\sum_{\mathrm{v}=1}^{\mathrm{n}} \mathrm{R}_{\mathrm{v}-1} \mathrm{a}_{\mathrm{v}}\left(\frac{\lambda_{\mathrm{n}}}{\mathrm{R}_{\mathrm{n}-1}}\right) \\
& =\frac{\mathrm{q}_{\mathrm{n}}}{\mathrm{Q}_{\mathrm{n}} \mathrm{Q}_{\mathrm{n}-1}} \sum_{\mathrm{v}=1}^{\mathrm{n}-1}\left(\mathrm{t}_{\mathrm{v}} \lambda_{\mathrm{v}} \mathrm{f}_{\mathrm{v}}+\frac{\mathrm{R}_{\mathrm{v}-1}}{\mathrm{r}_{\mathrm{v}}} \mathrm{p}_{\mathrm{v}} \mathrm{t}_{\mathrm{v}} \lambda_{\mathrm{v}} \mathrm{f}_{\mathrm{v}}+\frac{\mathrm{R}_{\mathrm{v}-1}}{\mathrm{r}_{\mathrm{v}}} \mathrm{t}_{\mathrm{v}} \Delta \lambda_{\mathrm{v}} \mathrm{F}_{\mathrm{v}+1}\right)+ \\
& +\frac{\mathrm{p}_{\mathrm{n}} \mathrm{q}_{\mathrm{n}} \mathrm{R}_{\mathrm{n}}}{\mathrm{Q}_{\mathrm{n}} \mathrm{Q}_{\mathrm{n}-1} \mathrm{r}_{\mathrm{n}}} \mathrm{t}_{\mathrm{n}} \mathrm{f}_{\mathrm{n}} \lambda_{\mathrm{n}}+ \\
& \quad+\frac{\mathrm{p}_{\mathrm{n}} \mathrm{q}_{\mathrm{n}}}{\mathrm{P}_{\mathrm{n}} \mathrm{Q}_{\mathrm{n}-1}}\left(\sum_{\mathrm{v}=1}^{\mathrm{n}-1}\left(\mathrm{t}_{\mathrm{v}} \lambda_{\mathrm{v}}+\frac{\mathrm{R}_{\mathrm{v}-1}}{\mathrm{r}_{\mathrm{v}}} \mathrm{t}_{\mathrm{v}} \Delta \lambda_{\mathrm{v}}\right)\right)+\frac{\mathrm{p}_{\mathrm{n}} \mathrm{q}_{\mathrm{n}} \mathrm{R}_{\mathrm{n}}}{\mathrm{P}_{\mathrm{n}} \mathrm{Q}_{\mathrm{n}-1} \mathrm{r}_{\mathrm{n}}} \mathrm{t}_{\mathrm{n}} \lambda_{\mathrm{n}} \\
& =\sum_{\mathrm{j}=1}^{7} \mathrm{~T}_{\mathrm{nj}}
\end{aligned}
$$

In order, to complete the proof, it is sufficient to show that-

$$
\sum_{n=1}^{\infty} \mathrm{n}^{\delta \mathrm{k}+\mathrm{k}-1}\left|\mathrm{~T}_{\mathrm{n}, \mathrm{j}}\right|^{\mathrm{k}}<\infty, \quad \mathrm{j}=1,2,3,4,5,6,7
$$

Now appling Hölder's inequality,

$$
\begin{aligned}
& \sum_{n=2}^{m+1} n^{\delta k+k-1}\left|T_{n, 1}\right|^{k}=\sum_{n=2}^{m+1} n^{\delta k+k-1}\left|\frac{q_{n}}{Q_{n} Q_{n-1}} \sum_{v=1}^{n-1}\left(t_{v} \lambda_{v} f_{v}\right)\right|^{k} \\
& \leq \sum_{\mathrm{n}=2}^{\mathrm{m}+1} \frac{\mathrm{n}^{\delta \mathrm{k}+\mathrm{k}-1} \mathrm{q}_{\mathrm{n}}^{\mathrm{k}}}{\mathrm{Q}_{\mathrm{n}}^{\mathrm{k}} \mathrm{Q}_{\mathrm{n}-1}} \sum_{\mathrm{v}=1}^{\mathrm{n}-1} \frac{1}{\mathrm{q}_{\mathrm{v}}^{\mathrm{k}-1}}\left|\mathrm{t}_{\mathrm{v}}\right|^{\mathrm{k}}\left|\lambda_{\mathrm{v}}\right|^{\mathrm{k}} \mathrm{F}_{\mathrm{v}}^{\mathrm{k}}\left(\sum_{\mathrm{v}=1}^{\mathrm{n}-1} \frac{\mathrm{q}_{\mathrm{v}}}{\mathrm{Q}_{\mathrm{n}-1}}\right)^{\mathrm{k}-1} \\
& =\square(1) \sum_{\mathrm{v}=1}^{\mathrm{m}} \frac{1}{\mathrm{q}_{\mathrm{v}}^{\mathrm{k}-1}}\left|\mathrm{t}_{\mathrm{v}}\right|^{\mathrm{k}}\left|\lambda_{\mathrm{v}}\right|^{\mathrm{k}} \mathrm{F}_{\mathrm{v}}^{\mathrm{k}} \sum_{\mathrm{n}=\mathrm{v}+1}^{\mathrm{m}+1} \frac{\mathrm{n}^{\delta \mathrm{k}+\mathrm{k}-1} \mathrm{q}_{\mathrm{n}}^{\mathrm{k}}}{\mathrm{Q}_{\mathrm{n}}^{\mathrm{k}} \mathrm{Q}_{\mathrm{n}-1}} \\
& =\square(1) \sum_{\mathrm{v}=1}^{\mathrm{m}} \mathrm{v}^{\delta \mathrm{k}+\mathrm{k}-1}\left|\mathrm{t}_{\mathrm{v}}\right|^{\mathrm{k}}\left|\lambda_{\mathrm{v}}\right|^{\mathrm{k}} \frac{\mathrm{F}_{\mathrm{v}}^{\mathrm{k}}}{\mathrm{Q}_{\mathrm{v}}^{\mathrm{k}}} \\
& =\square(1) \sum_{\mathrm{n}=2}^{\mathrm{m}+1} \mathrm{n}^{\delta \mathrm{k}+\mathrm{k}-1}\left|\mathrm{~T}_{\mathrm{n}, 2}\right|^{\mathrm{k}}=\sum_{\mathrm{n}=2}^{\mathrm{m}+1} \mathrm{n}^{\delta \mathrm{k}+\mathrm{k}-1}\left|\frac{\mathrm{q}_{\mathrm{n}}}{\mathrm{Q}_{\mathrm{n}} \mathrm{Q}_{\mathrm{n}-1}} \sum_{\mathrm{v}=1}^{\mathrm{n}-1}\left(\frac{\mathrm{R}_{\mathrm{v}-1} \mathrm{p}_{\mathrm{v}}}{\mathrm{r}_{\mathrm{v}}} \mathrm{t}_{\mathrm{v}} \lambda_{\mathrm{v}} \mathrm{f}_{\mathrm{v}}\right)\right|^{\mathrm{k}} \\
& \leq \sum_{n=2}^{m+1} \frac{n^{\delta k+k-1} q_{n}^{k}}{Q_{n}^{k} Q_{n-1}-1} \sum_{v=1}\left(\frac{R_{v}^{k} p_{v}^{k}}{q_{v}^{k-1}}\left|t_{v}\right|^{k}\left|\lambda_{v}\right|^{k} f_{v}^{k}\right)\left(\sum_{v=1}^{n-1} \frac{q_{v}}{Q_{n-1}}\right)^{k-1} \\
& =\square(1) \sum_{\mathrm{v}=1}^{\mathrm{m}}\left(\frac{\mathrm{R}_{\mathrm{v}}^{\mathrm{k}} \mathrm{p}_{\mathrm{v}}^{\mathrm{k}}}{\mathrm{q}_{\mathrm{v}}^{\mathrm{k}-1}}\left|\mathrm{t}_{\mathrm{v}}\right|^{\mathrm{k}}\left|\lambda_{\mathrm{v}}\right|^{\mathrm{k}} \mathrm{f}_{\mathrm{v}}^{\mathrm{k}}\right) \sum_{\mathrm{n}=\mathrm{v}+1}^{\mathrm{m}+1} \frac{\mathrm{n}^{\delta \mathrm{k}+\mathrm{k}-1} \mathrm{q}_{\mathrm{n}}^{\mathrm{k}}}{\mathrm{Q}_{\mathrm{n}}^{\mathrm{k}} \mathrm{Q}_{\mathrm{n}-1}} \\
& =\square(1) \sum_{\mathrm{v}=1}^{\mathrm{m}}\left(\mathrm{v}^{\delta \mathrm{k}+\mathrm{k}-1} \frac{\mathrm{R}_{\mathrm{v}}^{\mathrm{k}} \mathrm{p}_{\mathrm{v}}^{\mathrm{k}}}{\mathrm{Q}_{\mathrm{v}}^{\mathrm{k}}}\left|\mathrm{t}_{\mathrm{v}}\right|^{\mathrm{k}}\left|\lambda_{\mathrm{v}}\right|^{\mathrm{k}} \mathrm{f}_{\mathrm{v}}^{\mathrm{k}}\right) \\
& =\square(1) \sum_{\mathrm{v}=1}^{\mathrm{m}}\left(\mathrm{v}^{\delta \mathrm{k}+\mathrm{k}-1}\left|\mathrm{t}_{\mathrm{v}}\right|^{\mathrm{k}}\right) \\
& =\square(1)
\end{aligned}
$$




$$
\begin{aligned}
& \sum_{\mathrm{n}=2}^{\mathrm{m}+1} \mathrm{n}^{\delta \mathrm{k}+\mathrm{k}-1}\left|\mathrm{~T}_{\mathrm{n}, 3}\right|=\sum_{\mathrm{n}=2}^{\mathrm{m}+1} \mathrm{n}^{\delta \mathrm{k}+\mathrm{k}-1}\left|\frac{\mathrm{q}_{\mathrm{n}}}{\mathrm{Q}_{\mathrm{n}} \mathrm{Q}_{\mathrm{n}-1}} \sum_{\mathrm{v}=1}^{\mathrm{n}-1}\left(\frac{\mathrm{R}_{\mathrm{v}-1}}{\mathrm{r}_{\mathrm{v}}} \mathrm{t}_{\mathrm{v}} \Delta \lambda_{\mathrm{v}} \mathrm{F}_{\mathrm{v}+1}\right)\right|^{\mathrm{k}} \\
& \leq \sum_{n=1}^{m+1} \frac{n^{\delta k+k-1} q_{n}^{k}}{Q_{n}^{k} Q_{n-1}} \sum_{v=1}^{n-1}\left(\frac{R_{v}^{k}-1}{q_{v}^{k-1} r_{v}^{k}}\left|t_{v}\right|^{k}\left|\Delta \lambda_{v}\right|^{k} F_{v+1}^{k}\right)\left\{\sum_{v=1}^{n-1} \frac{q_{v}}{Q_{n-1}}\right\}^{k} \\
& =\square(1) \sum_{\mathrm{v}=1}^{\mathrm{m}}\left(\frac{\mathrm{R}_{\mathrm{v}-1}^{\mathrm{k}}}{\mathrm{q}_{\mathrm{v}}^{\mathrm{k}-1} \mathrm{r}_{\mathrm{v}}^{\mathrm{k}}}\left|\mathrm{t}_{\mathrm{v}}\right|^{\mathrm{k}}\left|\Delta \lambda_{\mathrm{v}}\right|^{\mathrm{k}} \mathrm{F}_{\mathrm{v}+1}^{\mathrm{k}}\right) \sum_{\mathrm{n}=\mathrm{v}+1}^{\mathrm{m}+1} \frac{\mathrm{n}^{\delta \mathrm{k}+\mathrm{k}-1} \mathrm{q}_{\mathrm{n}}^{\mathrm{k}}}{\mathrm{Q}_{\mathrm{n}}^{\mathrm{k}} \mathrm{Q}_{\mathrm{n}-1}} \\
& =\square(1) \sum_{\mathrm{v}=1}^{\mathrm{m}}\left(\mathrm{v}^{\delta \mathrm{k}+\mathrm{k}-1} \frac{\mathrm{R}_{\mathrm{v}-1}^{\mathrm{k}}}{\mathrm{Q}_{\mathrm{v}}^{\mathrm{k}-1} \mathrm{r}_{\mathrm{v}}^{\mathrm{k}}}\left|\mathrm{t}_{\mathrm{v}}\right|^{\mathrm{k}}\left|\Delta \lambda_{\mathrm{v}}\right|^{\mathrm{k}} \mathrm{F}_{\mathrm{v}+1}^{\mathrm{k}}\right) \\
& =\square(1)
\end{aligned}
$$

$$
\begin{aligned}
\sum_{n=2}^{m+1} n^{\delta k+k-1}\left|T_{n, 4}\right| & =\sum_{n=1}^{m} n^{\delta k+k-1}\left|\frac{p_{n} q_{n} R_{n}}{Q_{n} Q_{n-1} r_{n}} t_{n} \lambda_{n} f_{n}\right|^{k} \\
= & \square(1) \sum_{n=1}^{m} n^{\delta k+k-1} \frac{p_{n}^{k} q_{n}^{k} R_{n}^{k}}{Q_{n}^{k} Q_{n-1}^{k} r_{n}^{k}}\left|\lambda_{n}\right|^{k}\left|t_{n}\right|^{k}\{u \operatorname{sing}(2.8)\} \\
= & (1)
\end{aligned}
$$

$$
\begin{aligned}
\sum_{\mathrm{n}=2}^{\mathrm{m}+1} \mathrm{n}^{\delta \mathrm{k}+\mathrm{k}-1}\left|\mathrm{~T}_{\mathrm{n}, 5}\right| & =\sum_{\mathrm{n}=2}^{\mathrm{m}+1} \mathrm{n}^{\delta \mathrm{k}+\mathrm{k}-1}\left|\frac{\mathrm{p}_{\mathrm{n}} \mathrm{q}_{\mathrm{n}}}{\mathrm{P}_{\mathrm{n}} \mathrm{Q}_{\mathrm{n}-1}} \sum_{\mathrm{v}=1}^{\mathrm{n}-1} \mathrm{t}_{\mathrm{v}} \lambda_{\mathrm{v}}\right|^{\mathrm{k}} \\
& \leq \sum_{\mathrm{n}=1}^{\mathrm{m}+1} \mathrm{n}^{\delta \mathrm{k}+\mathrm{k}-1} \frac{\mathrm{p}_{\mathrm{n}}^{\mathrm{k}} \mathrm{q}_{\mathrm{n}}^{\mathrm{k}}}{\mathrm{P}_{\mathrm{n}}^{\mathrm{k}} \mathrm{Q}_{\mathrm{n}-1}} \sum_{\mathrm{v}=1}^{\mathrm{n}-1}\left|\mathrm{t}_{\mathrm{v}}\right|^{\mathrm{k}}\left|\lambda_{\mathrm{v}}\right|^{\mathrm{k}} \frac{1}{\mathrm{q}_{\mathrm{v}}^{\mathrm{k}-1}}\left\{\sum_{\mathrm{v}=1}^{\mathrm{n}-1} \frac{\mathrm{q}_{\mathrm{v}}}{\mathrm{Q}_{\mathrm{v}-1}}\right\}^{\mathrm{k}-1} \\
& =\square(1) \sum_{\mathrm{v}=1}^{\mathrm{m}}\left|\mathrm{t}_{\mathrm{v}}\right|^{\mathrm{k}}\left|\lambda_{\mathrm{v}}\right|^{\mathrm{k}} \frac{1}{\mathrm{q}_{\mathrm{v}}^{\mathrm{k}-1}} \sum_{\mathrm{n}=\mathrm{v}+1}^{\mathrm{m}+1} \mathrm{n}^{\delta \mathrm{k}+\mathrm{k}-1} \frac{\mathrm{p}_{\mathrm{n}}^{\mathrm{k}} \mathrm{q}_{\mathrm{n}}^{\mathrm{k}}}{\mathrm{P}_{\mathrm{n}}^{\mathrm{k}} \mathrm{Q}_{\mathrm{n}-1}} \\
& =\square(1) \sum_{\mathrm{v}=1}^{\mathrm{m}}\left|\mathrm{t}_{\mathrm{v}}\right|^{\mathrm{k}}\left|\lambda_{\mathrm{v}}\right|^{\mathrm{k}} \frac{1}{\mathrm{q}_{\mathrm{v}}^{\mathrm{k}-1}} \sum_{\mathrm{n}=\mathrm{v}+1}^{\mathrm{m}+1} \frac{\mathrm{n}^{\delta \mathrm{k}+\mathrm{k}-1} \mathrm{q}_{\mathrm{n}}^{\mathrm{k}}}{\mathrm{Q}_{\mathrm{n}}^{\mathrm{k}} \mathrm{Q}_{\mathrm{n}-1}} \\
& =\square(1) \sum_{\mathrm{v}=1}^{\mathrm{m}} \mathrm{v}^{\delta \mathrm{k}+\mathrm{k}-1}\left|\mathrm{t}_{\mathrm{v}}\right|^{\mathrm{k}}\left|\lambda_{\mathrm{v}}\right|^{\mathrm{k}} \frac{1}{\mathrm{Q}_{\mathrm{v}}^{\mathrm{k}}} \quad\{\text { using (2.3)\}} \\
& =\square(1) \quad\{\text { using (2.6)\}}
\end{aligned}
$$

$$
\begin{aligned}
& \sum_{\mathrm{n}=2}^{\mathrm{m}+1} \mathrm{n}^{\delta \mathrm{k}+\mathrm{k}-1}\left|\mathrm{~T}_{\mathrm{n}, 6}\right|=\sum_{\mathrm{n}=2}^{\mathrm{m}+1} \mathrm{n}^{\delta \mathrm{k}+\mathrm{k}-1}\left|\frac{\mathrm{p}_{\mathrm{n}} \mathrm{q}_{\mathrm{n}}}{\mathrm{P}_{\mathrm{n}} \mathrm{Q}_{\mathrm{n}-1}} \sum_{\mathrm{v}=1}^{\mathrm{n}-1} \frac{\mathrm{R}_{\mathrm{v}-1}}{\mathrm{r}_{\mathrm{v}}} \mathrm{t}_{\mathrm{v}} \Delta \lambda_{\mathrm{v}}\right|^{\mathrm{k}} \\
& \leq \sum_{\mathrm{n}=1}^{\mathrm{m}+1} \mathrm{n}^{\delta \mathrm{k}+\mathrm{k}-1} \frac{\mathrm{p}_{\mathrm{n}}^{\mathrm{k}} \mathrm{q}_{\mathrm{n}}^{\mathrm{k}}}{\mathrm{P}_{\mathrm{n}}^{\mathrm{k}} \mathrm{Q}_{\mathrm{n}-1}} \sum_{\mathrm{v}=1}^{\mathrm{n}-1}\left|\mathrm{t}_{\mathrm{v}}\right|^{\mathrm{k}}\left|\Delta \lambda_{\mathrm{v}}\right|^{\mathrm{k}} \frac{\mathrm{R}_{\mathrm{v}-1}^{\mathrm{k}}}{\mathrm{q}_{\mathrm{v}}^{\mathrm{k}-1} \mathrm{r}_{\mathrm{v}}^{\mathrm{k}}}\left\{\sum_{\mathrm{v}=1}^{\mathrm{n}-1} \frac{\mathrm{q}_{\mathrm{v}}}{\mathrm{Q}_{\mathrm{v}-1}}\right\}^{\mathrm{k}-1} \\
&=(1) \sum_{\mathrm{v}=1}^{\mathrm{m}}\left|\mathrm{t}_{\mathrm{v}}\right|^{\mathrm{k}}\left|\Delta \lambda_{\mathrm{v}}\right|^{\mathrm{k}} \frac{\mathrm{R}_{\mathrm{v}-1}^{\mathrm{k}}}{\mathrm{q}_{\mathrm{v}}^{\mathrm{k}-1} \mathrm{r}_{\mathrm{v}}^{\mathrm{k}}} \sum_{\mathrm{n}=\mathrm{v}+1}^{\mathrm{m}+1} \mathrm{n}^{\delta \mathrm{k}+\mathrm{k}-1} \frac{\mathrm{p}_{\mathrm{n}}^{\mathrm{k}} \mathrm{q}_{\mathrm{n}}^{\mathrm{k}}}{\mathrm{P}_{\mathrm{n}}^{\mathrm{k}} \mathrm{Q}_{\mathrm{n}-1}}
\end{aligned}
$$




$$
\begin{aligned}
& =\square(1) \sum_{\mathrm{v}=1}^{\mathrm{m}} \mathrm{v}^{\delta \mathrm{k}+\mathrm{k}-1}\left|\mathrm{t}_{\mathrm{v}}\right|^{\mathrm{k}}\left|\Delta \lambda_{\mathrm{v}}\right|^{\mathrm{k}} \frac{\mathrm{R}_{\mathrm{v}-1}^{\mathrm{k}}}{\mathrm{Q}_{\mathrm{v}}^{\mathrm{k}-1} \mathrm{r}_{\mathrm{v}}^{\mathrm{k}}} \\
& =\square(1) \quad\{\text { Using (2.11) }\}
\end{aligned}
$$

Finally,

$$
\begin{aligned}
\sum_{n=1}^{m} n^{\delta k+k-1}\left|T_{n, 7}\right| & =\sum_{n=1}^{m} n^{\delta k+k-1}\left|\frac{p_{n} q_{n} R_{n}}{P_{n} Q_{n-1} r_{n}} t_{n} \lambda_{n}\right|^{k} \\
& =\square(1) \sum_{n=1}^{m} n^{\delta k+k-1}\left|t_{n}\right|^{k}\left|\lambda_{n}\right|^{k}\left(\frac{p_{n} q_{n} R_{n}}{P_{n} Q_{n} r_{n}}\right)^{k}\{\text { using 2.9 } \\
= & (1)
\end{aligned}
$$

This completes the proof of the THEOREM 1.

\section{PROOF OF THE THEOREM 2 :}

For $\mathrm{k} \geq 1, \delta \geq 0$, define-

$$
\begin{aligned}
& \mathrm{A}^{*}=\left\{\left(\mathrm{a}_{\mathrm{j}}\right) ; \sum \mathrm{a}_{\mathrm{j}} \text { is summable }\left|\mathrm{R}, \mathrm{r}_{\mathrm{n}}, \delta\right|_{\mathrm{k}}\right\} \\
& \mathrm{B}^{*}=\left\{\left(\mathrm{b}_{\mathrm{j}}\right) ; \sum \mathrm{b}_{\mathrm{j}} \lambda_{\mathrm{j}} \text { is summable }\left|\left(\mathrm{R}, \mathrm{q}_{\mathrm{n}}\right)\left(\mathrm{R}, \mathrm{p}_{\mathrm{n}}\right), \delta\right|_{\mathrm{k}}\right\}
\end{aligned}
$$

from (3.3), we have-

$$
\mathrm{T}_{\mathrm{n}}=\sum_{\mathrm{v}=1}^{\mathrm{n}}\left(\frac{\mathrm{q}_{\mathrm{n}} \mathrm{F}_{\mathrm{v}}}{\mathrm{Q}_{\mathrm{n}} \mathrm{Q}_{\mathrm{n}-1}}+\frac{\mathrm{p}_{\mathrm{n}} \mathrm{q}_{\mathrm{n}}}{\mathrm{P}_{\mathrm{n}} \mathrm{Q}_{\mathrm{n}-1}}\right) \mathrm{a}_{\mathrm{v}} \lambda_{\mathrm{v}}
$$

with $t_{n}$ and $T_{n}$ as defined by (2.12) and (3.8), the space $A^{*}$ and $B^{*}$ BK-spaces with norms defined by-

$$
\begin{aligned}
& \|\mathrm{c}\|_{1}=\left\{\left|\mathrm{t}_{0}\right|^{\mathrm{k}}+\sum_{\mathrm{n}=1}^{\infty} \mathrm{n}^{\delta \mathrm{k}+\mathrm{k}-1}\left|\mathrm{t}_{\mathrm{n}}\right|^{\mathrm{k}}\right\}^{1 / \mathrm{k}} \\
& \|\mathrm{c}\|_{2}=\left\{\left|\mathrm{T}_{0}\right|^{\mathrm{k}}+\sum_{\mathrm{n}=1}^{\infty} \mathrm{n}^{\delta \mathrm{k}+\mathrm{k}-1}\left|\mathrm{~T}_{\mathrm{n}}\right|^{\mathrm{k}}\right\}^{1 / \mathrm{k}}
\end{aligned}
$$

respectively, by the hypothesis of the theorem,

$$
\|\mathrm{c}\|_{1}<\infty \Rightarrow\|\mathrm{c}\|_{2}<\infty
$$

The inclusion map i; $\mathrm{A}^{*} \rightarrow \mathrm{B}^{*}$ defined by $\mathrm{i}(\mathrm{a})=\mathrm{a}$ is continuous since $\mathrm{A}^{*}$ and $\mathrm{B}^{*}$ are $\mathrm{BK}$ spaces. By the closed graph theorem, there exists a constant, $\mathrm{K}>0$, such that-

$$
\|\mathrm{c}\|_{2} \leq \mathrm{K}\|\mathrm{c}\|_{1}
$$

Let $e_{n}$ denote the $n^{\text {th }}$ coordinate vector, from (3.4) and (3.8) with $\left\{a_{n}\right\}$ defined by $a_{n}=e_{n}-e_{n+1}$, $\mathrm{n}=\mathrm{v}, \mathrm{a}_{\mathrm{n}}=0$, otherwise, we have 


$$
\begin{aligned}
& \mathrm{t}_{\mathrm{n}}= \begin{cases}0, & \mathrm{n}<\mathrm{v} \\
\frac{\mathrm{r}_{\mathrm{v}},}{\mathrm{R}_{\mathrm{v}}} & \mathrm{n}=\mathrm{v} \\
-\frac{\mathrm{r}_{\mathrm{n}} \mathrm{r}_{\mathrm{v}}}{\mathrm{R}_{\mathrm{n}} \mathrm{R}_{\mathrm{n}-1}}, & \mathrm{n}>\mathrm{v}\end{cases} \\
& \mathrm{T}_{\mathrm{n}}=\left\{\begin{array}{l}
\left(\frac{\mathrm{q}_{\mathrm{v}} \mathrm{F}_{\mathrm{v}}}{\mathrm{Q}_{\mathrm{v}} \mathrm{Q}_{\mathrm{v}-1}}+\frac{\mathrm{p}_{\mathrm{v}} \mathrm{q}_{\mathrm{v}}}{\mathrm{P}_{\mathrm{v}} \mathrm{Q}_{\mathrm{v}-1}}\right) \lambda_{\mathrm{v}}, \mathrm{n}=\mathrm{v} \\
\Delta_{\mathrm{v}}\left(\frac{\mathrm{q}_{\mathrm{n}} \mathrm{F}_{\mathrm{v}}}{\mathrm{Q}_{\mathrm{n}} \mathrm{Q}_{\mathrm{n}-1}}+\frac{\mathrm{p}_{\mathrm{n}} \mathrm{q}_{\mathrm{n}}}{\mathrm{P}_{\mathrm{n}} \mathrm{Q}_{\mathrm{n}-1}}\right) \lambda_{\mathrm{v}}, \mathrm{n}>\mathrm{v}
\end{array}\right.
\end{aligned}
$$

From (3.9), we have -

$$
\|c\|_{1}=\left\{v^{\delta k+k-1}\left(\frac{q_{v}}{Q_{v}}\right)^{k}+\sum_{n=v+1}^{\infty} n^{\delta k+k-1}\left(\frac{q_{n} q_{v}}{Q_{n} Q_{n-1}}\right)^{k}\right\}^{1 / k}
$$

$$
\begin{aligned}
\|c\|_{2}=\left\{v^{\delta k+k-1}\left|\left(\frac{q_{v} F_{v}}{Q_{v} Q_{v-1}}+\frac{p_{v} q_{v}}{P_{v} Q_{v-1}}\right) \lambda_{v}\right|^{k}+\right. \\
\left.+\sum_{n=v+1}^{\infty} n^{\delta k+k-1}\left|\Delta_{v}\left(\frac{q_{n} F_{v}}{Q_{n} Q_{n-1}}+\frac{p_{n} q_{n}}{P_{n} Q_{n-1}}\right) \lambda_{v}\right|^{k}\right\}^{1 / k}
\end{aligned}
$$

Applying (3.11), we obtain-

$$
\begin{aligned}
& \left\{v^{\delta k+k-1}\left|\left(\frac{q_{v} F_{v}}{Q_{v} Q_{v-1}}+\frac{p_{v} q_{v}}{P_{v} Q_{v-1}}\right) \lambda_{v}\right|^{k}+\right. \\
& \left.\quad+\sum_{n=v+1}^{\infty} n^{\delta k+k-1}\left|\Delta_{v}\left(\frac{q_{n} F_{v}}{Q_{n} Q_{n-1}}+\frac{p_{n} q_{n}}{P_{n} Q_{n-1}}\right) \lambda_{v}\right|^{k}\right\} \\
& =\square(1)\left\{v^{\delta k+k-1}\left(\frac{r_{v}}{R_{v}}\right)^{k}+\sum_{n=v+1}^{\infty} n^{\delta k+k-1}\left(\frac{r_{n} r_{v}}{R_{n} R_{n-1}}\right)^{k}\right\}
\end{aligned}
$$

As the right hand side of (3.13), by (2.3) is

$$
=\square(1)\left\{\mathrm{v}^{\delta \mathrm{k}+\mathrm{k}-1}\left(\frac{\mathrm{r}_{\mathrm{v}}}{\mathrm{R}_{\mathrm{v}}}\right)^{\mathrm{k}}+\frac{\mathrm{r}_{\mathrm{v}}^{\mathrm{k}}}{\mathrm{R}_{\mathrm{v}}^{\mathrm{k}-1}} \sum_{\mathrm{n}=\mathrm{v}+1}^{\infty} \mathrm{n}^{\delta \mathrm{k}+\mathrm{k}-1}\left(\frac{\mathrm{r}_{\mathrm{n}}^{\mathrm{k}}}{\mathrm{R}_{\mathrm{n}}^{\mathrm{k}} \mathrm{R}_{\mathrm{n}-1}}\right)\right\}
$$




$$
\begin{aligned}
& =\square(1)\left\{\mathrm{v}^{\delta \mathrm{k}+\mathrm{k}-1}\left(\frac{\mathrm{r}_{\mathrm{v}}}{\mathrm{R}_{\mathrm{v}}}\right)^{\mathrm{k}}+\left(\frac{\mathrm{r}_{\mathrm{v}}}{\mathrm{R}_{\mathrm{v}}}\right)^{\mathrm{k}-1} \mathrm{n}^{\delta \mathrm{k}+\mathrm{k}-1}\left(\frac{\mathrm{r}_{\mathrm{v}}}{\mathrm{R}_{\mathrm{v}}}\right)^{\mathrm{k}}\right\} \\
& =\square(1)\left\{\mathrm{v}^{\delta \mathrm{k}+\mathrm{k}-1}\left(\frac{\mathrm{r}_{\mathrm{v}}}{\mathrm{R}_{\mathrm{v}}}\right)^{\mathrm{k}}\right\}
\end{aligned}
$$

And the fact that each term of the left hand side of (3.13) is

$$
\begin{aligned}
& \square(1)\left\{\mathrm{v}^{\delta \mathrm{k}+\mathrm{k}-1}\left(\frac{\mathrm{r}_{\mathrm{v}}}{\mathrm{R}_{\mathrm{v}}}\right)^{\mathrm{k}}\right\} \text {, we obtain- } \\
& \left\{\mathrm{v}^{\delta \mathrm{k}+\mathrm{k}-1}\left(\frac{\mathrm{q}_{\mathrm{v}} \mathrm{F}_{\mathrm{v}}}{\mathrm{Q}_{\mathrm{v}} \mathrm{Q}_{\mathrm{v}-1}}+\frac{\mathrm{p}_{\mathrm{v}} \mathrm{q}_{\mathrm{v}}}{\mathrm{P}_{\mathrm{v}} \mathrm{Q}_{\mathrm{v}-1}}\right)^{\mathrm{k}}\left|\lambda_{\mathrm{v}}\right|^{\mathrm{k}}\right\}=\square\left\{\mathrm{v}^{\delta \mathrm{k}+\mathrm{k}-1}\left(\frac{\mathrm{r}_{\mathrm{v}}}{\mathrm{R}_{\mathrm{v}}}\right)^{\mathrm{k}}\right\}
\end{aligned}
$$

Which implies by (3.3)-

$$
\left(\frac{\mathrm{q}_{\mathrm{v}}}{\mathrm{Q}_{\mathrm{v}} \mathrm{Q}_{\mathrm{v}-1}}\right)^{\mathrm{k}}\left(1+\mathrm{F}_{\mathrm{v}}\right)^{\mathrm{k}}\left|\lambda_{\mathrm{v}}\right|^{\mathrm{k}}=\square\left(\frac{\mathrm{r}_{\mathrm{v}}}{\mathrm{R}_{\mathrm{v}}}\right)^{\mathrm{k}}
$$

that is -

$$
\left|\lambda_{\mathrm{v}}\right|=\square\left(\frac{\mathrm{Q}_{\mathrm{v}} \mathrm{Q}_{\mathrm{v}-1} \mathrm{r}_{\mathrm{v}}}{\left(1+\mathrm{F}_{\mathrm{v}}\right) \mathrm{q}_{\mathrm{v}} \mathrm{R}_{\mathrm{v}}}\right)
$$

Also, we have by (3.13)

$$
\begin{aligned}
& \sum_{\mathrm{n}=\mathrm{v}+1}^{\infty} \mathrm{n}^{\delta \mathrm{k}+\mathrm{k}-1}\left|\left(\frac{\mathrm{q}_{\mathrm{n}} \mathrm{p}_{\mathrm{v}} \mathrm{f}_{\mathrm{v}}}{\mathrm{Q}_{\mathrm{n}} \mathrm{Q}_{\mathrm{n}-1}}\right) \lambda_{\mathrm{v}}+\left(\frac{\mathrm{q}_{\mathrm{n}} \mathrm{F}_{\mathrm{v}+1}}{\mathrm{Q}_{\mathrm{n}} \mathrm{Q}_{\mathrm{n}-1}}+\frac{\mathrm{p}_{\mathrm{n}} \mathrm{q}_{\mathrm{n}}}{\mathrm{P}_{\mathrm{n}} \mathrm{Q}_{\mathrm{n}-1}}\right) \Delta \lambda_{\mathrm{v}}\right|^{\mathrm{k}} \\
& =\square\left\{\mathrm{v}^{\delta \mathrm{k}+\mathrm{k}-1}\left(\frac{\mathrm{r}_{\mathrm{v}}}{\mathrm{R}_{\mathrm{v}}}\right)^{\mathrm{k}}\right\}
\end{aligned}
$$

The above, via the linear independence of $\lambda_{\mathrm{v}}$ and $\Delta \lambda_{\mathrm{v}}$ implies-

$$
\begin{aligned}
& \sum_{n=v+1}^{\infty} n^{\delta k+k-1}\left(\frac{q_{n} F_{v+1}}{Q_{n} Q_{n-1}}+\frac{p_{n} q_{n}}{P_{n} Q_{n-1}}\right)^{k}\left|\Delta \lambda_{v}\right|^{k}=\square\left\{v^{\delta k+k-1}\left(\frac{q_{v}}{Q_{v}}\right)^{k}\right\} \\
& \left|\Delta \lambda_{v}\right|^{k}\left(1+F_{v+1}\right)^{k} \sum_{n=v+1}^{\infty} n^{\delta k+k-1}\left(\frac{q_{n}}{Q_{n} Q_{n-1}}\right)^{k}=\square\left\{v^{\delta k+k-1}\left(\frac{q_{v}}{Q_{v}}\right)^{k}\right\}
\end{aligned}
$$

As by (3.4), via the mean value theorem, 


$$
\begin{aligned}
& \frac{1}{\mathrm{Q}_{\mathrm{v}}^{\mathrm{k}}}=\sum_{\mathrm{n}=\mathrm{v}+1}^{\infty} \Delta\left(\frac{1}{\mathrm{Q}_{\mathrm{n}-1}^{\mathrm{k}}}\right)=\square(1) \sum_{\mathrm{n}=\mathrm{v}+1}^{\infty} \frac{\left|\Delta \mathrm{Q}_{\mathrm{n}-1}^{\mathrm{k}}\right|}{\mathrm{Q}_{\mathrm{n}-1}^{\mathrm{k}} \mathrm{Q}_{\mathrm{n}}^{\mathrm{k}}}=\square(1) \sum_{\mathrm{n}=\mathrm{v}+1}^{\infty} \frac{\mathrm{Q}_{\mathrm{n}-1}^{\mathrm{k}-1} \mathrm{q}_{\mathrm{n}}}{\mathrm{Q}_{\mathrm{n}-1}^{\mathrm{k}} \mathrm{Q}_{\mathrm{n}}^{\mathrm{k}}} \\
& =\square(1) \sum_{\mathrm{n}=\mathrm{v}+1}^{\infty} \mathrm{n}^{\delta \mathrm{k}+\mathrm{k}-1}\left(\frac{\mathrm{q}_{\mathrm{n}}}{\mathrm{Q}_{\mathrm{n}-1} \mathrm{Q}_{\mathrm{n}}}\right)^{\mathrm{k}}
\end{aligned}
$$

Then,

$$
\left|\Delta \lambda_{\mathrm{v}}\right|^{\mathrm{k}}\left(1+\mathrm{F}_{\mathrm{v}+1}\right)^{\mathrm{k}}\left(\frac{1}{\mathrm{Q}_{\mathrm{v}}^{\mathrm{k}}}\right)=\square\left\{\mathrm{v}^{\delta \mathrm{k}+\mathrm{k}-1}\left(\frac{\mathrm{r}_{\mathrm{v}}}{\mathrm{R}_{\mathrm{v}}}\right)^{\mathrm{k}}\right\}
$$

Which implies

$$
\lambda_{\mathrm{v}}=\square\left\{\frac{\mathrm{v}^{(\delta+1)-1 / \mathrm{k}} \mathrm{r}_{\mathrm{v}} \mathrm{Q}_{\mathrm{v}}}{\left(1+\mathrm{F}_{\mathrm{v}+1}\right) \mathrm{R}_{\mathrm{v}}}\right\}
$$

Also by (3.14)

$$
\begin{aligned}
& \sum_{\mathrm{n}=\mathrm{v}+1}^{\infty} \mathrm{n}^{\delta \mathrm{k}+\mathrm{k}-1}\left|\left(\frac{\mathrm{q}_{\mathrm{n}} \mathrm{p}_{\mathrm{v}} \mathrm{f}_{\mathrm{v}}}{\mathrm{Q}_{\mathrm{n}} \mathrm{Q}_{\mathrm{n}-1}} \lambda_{\mathrm{v}}\right)\right|^{\mathrm{k}}=\square\left\{\mathrm{v}^{\delta \mathrm{k}+\mathrm{k}-1}\left(\frac{\mathrm{r}_{\mathrm{v}}}{\mathrm{R}_{\mathrm{v}}}\right)^{\mathrm{k}}\right\} \\
& \mathrm{p}_{\mathrm{v}}^{\mathrm{k}} \mathrm{f}_{\mathrm{v}}^{\mathrm{k}}\left|\lambda_{\mathrm{v}}\right|^{\mathrm{k}} \sum_{\mathrm{n}=\mathrm{v}+1}^{\infty} \mathrm{n}^{\delta \mathrm{k}+\mathrm{k}-1}\left(\frac{\mathrm{q}_{\mathrm{n}}}{\mathrm{Q}_{\mathrm{n}} \mathrm{Q}_{\mathrm{n}-1}}\right)^{\mathrm{k}}=\square\left\{\mathrm{v}^{\delta \mathrm{k}+\mathrm{k}-1}\left(\frac{\mathrm{r}_{\mathrm{v}}}{\mathrm{R}_{\mathrm{v}}}\right)^{\mathrm{k}}\right\} \\
& \mathrm{p}_{\mathrm{v}}^{\mathrm{k}} \mathrm{f}_{\mathrm{v}}^{\mathrm{k}}\left|\lambda_{\mathrm{v}}\right|^{\mathrm{k}}\left(\frac{1}{\mathrm{Q}_{\mathrm{v}}^{\mathrm{k}}}\right)=\square\left\{\mathrm{v}^{\delta \mathrm{k}+\mathrm{k}-1}\left(\frac{\mathrm{r}_{\mathrm{v}}}{\mathrm{R}_{\mathrm{v}}}\right)^{\mathrm{k}}\right\}
\end{aligned}
$$

Which implies

$$
\lambda_{\mathrm{v}}=\square\left\{\mathrm{v}^{(\delta+1)-1 / \mathrm{k}}\left(\frac{\mathrm{r}_{\mathrm{v}} \mathrm{Q}_{\mathrm{v}}}{\mathrm{p}_{\mathrm{v}} \mathrm{f}_{\mathrm{v}} \mathrm{R}_{\mathrm{v}}}\right)\right\}
$$

This completes the proof of the THEOREM 2 .

\section{Corollaries}

Cor. 1

Let $\mathrm{k} \geq 1$, define-

$$
\mathrm{f}_{\mathrm{v}}=\sum_{\mathrm{r}=\mathrm{v}}^{\mathrm{n}} \frac{\mathrm{q}_{\mathrm{r}}}{\mathrm{r}}, \quad \mathrm{F}_{\mathrm{v}}=\sum_{\mathrm{r}=\mathrm{v}}^{\mathrm{n}} \mathrm{f}_{\mathrm{r}}
$$

Let $\quad \mathrm{n}=\square\left(\mathrm{Q}_{\mathrm{n}}\right)$

Then sufficient conditions for the implication,

$\sum \mathrm{a}_{\mathrm{n}}$ is summable $|\mathrm{C}, 1, \delta|_{\mathrm{k}} \Rightarrow \sum \mathrm{a}_{\mathrm{n}} \lambda_{\mathrm{n}}$ is summable $\left|\left(\mathrm{R}, \mathrm{q}_{\mathrm{n}}\right)(\mathrm{C}, 1), \delta\right|_{\mathrm{k}}$

are (2.5), (2.6) and the following-

$$
\begin{aligned}
& \mathrm{v}\left|\lambda_{\mathrm{v}}\right|=\square\left(\mathrm{Q}_{\mathrm{v}}\right) \\
& \mathrm{vq}_{\mathrm{v}}\left|\lambda_{\mathrm{v}}\right|=\square\left(\mathrm{Q}_{\mathrm{v}} \mathrm{Q}_{\mathrm{v}-1}\right) \\
& \mathrm{nq}_{\mathrm{n}}\left|\lambda_{\mathrm{n}}\right|=\square\left(\mathrm{nQ}_{\mathrm{n}}\right)
\end{aligned}
$$




$$
\begin{aligned}
& \mathrm{v}\left|\Delta \lambda_{\mathrm{v}}\right| \mathrm{F}_{\mathrm{v}+1}=\square\left(\mathrm{Q}_{\mathrm{v}}\right) \\
& \left|\Delta \lambda_{\mathrm{v}}\right|=\square\left(\mathrm{q}_{\mathrm{v}}\right) \\
& \mathrm{v}\left|\Delta \lambda_{\mathrm{v}}\right|=\square\left(\mathrm{Q}_{\mathrm{v}}\right)
\end{aligned}
$$

Proof: The proof follows from Theorem 1, by putting

$$
\mathrm{p}_{\mathrm{n}}=\mathrm{r}_{\mathrm{n}}=1 \text { for all } \mathrm{n} \text {. }
$$

Cor. 2 If $\delta=0$, then above corollary reduces to Cor. (2.1) of SULAIMAN[3].

Cor. 3 Let $\mathrm{k} \geq 1$, define-

$$
\mathrm{f}_{\mathrm{v}}=\sum_{\mathrm{r}=\mathrm{v}}^{\mathrm{n}} \frac{1}{\mathrm{P}_{\mathrm{r}}}, \quad \mathrm{F}_{\mathrm{v}}=\sum_{\mathrm{r}=\mathrm{v}}^{\mathrm{n}} \mathrm{p}_{\mathrm{r}} \mathrm{f}_{\mathrm{r}}
$$

Let (2.2) be satisfied, then sufficient conditions for the implication,

$\sum \mathrm{a}_{\mathrm{n}}$ is summable $|\mathrm{C}, 1, \delta|_{\mathrm{k}} \Rightarrow \sum \mathrm{a}_{\mathrm{n}} \lambda_{\mathrm{n}}$ is summable $\left|(\mathrm{C}, 1)\left(\mathrm{R}, \mathrm{p}_{\mathrm{n}}\right), \delta\right|_{\mathrm{k}}$

$$
\begin{array}{ll}
\text { are, } & \left|\lambda_{\mathrm{v}}\right| \mathrm{F}_{\mathrm{v}}=\square(\mathrm{v}) \\
& \left|\lambda_{\mathrm{v}}\right|=\square(\mathrm{n}) \\
& \mathrm{p}_{\mathrm{v}} \lambda_{\mathrm{v}}=\square(1) \\
& \left|\Delta \lambda_{\mathrm{v}}\right| \mathrm{F}_{\mathrm{v}+1}=\square(1) \\
& \left|\Delta \lambda_{\mathrm{v}}\right|=\square(1)
\end{array}
$$

Proof:The proof follows from Theorem 1 by putting $q_{n}=r_{n}=1$, for all $n$, noticing that (2.3) is satisfied as-

$$
\sum_{n=v+1}^{\infty} \frac{1}{n(n-1)}=\sum_{n=v+1}^{\infty}\left[\frac{1}{(n-1)}-\frac{1}{n}\right]=\frac{1}{v}
$$

Cor. 4

If $\delta=0$, then above corollary reduces to Cor. (2.2) of SULAIMAN[3].

Cor. 5

Let $\mathrm{f}_{\mathrm{v}}, \mathrm{F}_{\mathrm{v}}$ be as defined in (4.1), let (2.3) and (4.2) be satisfied, then sufficient conditions for implications

$\sum \mathrm{a}_{\mathrm{n}}$ is summable $\left|\mathrm{R}, \mathrm{r}_{\mathrm{n}}, \delta\right|_{\mathrm{k}} \Rightarrow \sum \mathrm{a}_{\mathrm{n}} \lambda_{\mathrm{n}}$ is summable $\left|\left(\mathrm{R}, \mathrm{q}_{\mathrm{n}}\right)(\mathrm{C}, 1), \delta\right|_{\mathrm{k}}$ are,

(2.5), (2.6), (2.10), (2.11) and the followings-

$$
\begin{aligned}
& \mathrm{R}_{\mathrm{v}}\left|\lambda_{\mathrm{v}}\right|=\square\left(\mathrm{Q}_{\mathrm{v}}\right) \\
& \mathrm{q}_{\mathrm{v}} \mathrm{R}_{\mathrm{v}}\left|\lambda_{\mathrm{v}}\right|=\square\left(\mathrm{Q}_{\mathrm{v}} \mathrm{Q}_{\mathrm{v}-1} \mathrm{r}_{\mathrm{v}}\right) \\
& \mathrm{q}_{\mathrm{n}} \mathrm{R}_{\mathrm{n}}\left|\lambda_{\mathrm{n}}\right|=\square\left(\mathrm{nQ}_{\mathrm{n}} \mathrm{r}_{\mathrm{n}}\right)
\end{aligned}
$$

Proof: The proof follows from Theorem 1

$$
\text { by putting } \mathrm{p}_{\mathrm{n}}=1 \forall \mathrm{n} \text {. }
$$

Cor. 6 If we put $\delta=0$, then above corollary reduces to Cor. (2.3) of SULAIMAN[3].

Cor. 7 Let $f_{v}$ and $F_{v}$ be defined in (4.1), let (4.3), (2.12) be satisfied and

$$
\mathrm{v}=\square\left(\mathrm{Q}_{\mathrm{v}}\right)
$$

then, necessary conditions for the implication (4.3) are,

$$
\lambda_{\mathrm{v}}=\square\left(\frac{\mathrm{Q}_{\mathrm{v}} \mathrm{Q}_{\mathrm{v}-1}}{\left(1+\mathrm{F}_{\mathrm{v}}\right) \mathrm{vq}_{\mathrm{v}}}\right), \quad \lambda_{\mathrm{v}}=\square\left(\frac{\mathrm{Q}_{\mathrm{v}}}{\mathrm{v}^{(1 / \mathrm{k}+\delta)} \mathrm{f}_{\mathrm{v}}}\right)
$$




$$
\Delta \lambda_{\mathrm{v}}=\square\left(\frac{\mathrm{Q}_{\mathrm{v}}}{\mathrm{v}^{(1 / \mathrm{k}+\delta)}\left(1+\mathrm{F}_{\mathrm{v}+1}\right)}\right)
$$

Proof: The proof follows from Theorem 2, by putting $p_{n}=r_{n}=1$ for all $n$.

Cor. 8

By putting $\delta=0$, corollary.7 reduces to Cor. 2.4 of SULAIMAN[3] .

Cor. 9

Let $\mathrm{f}_{\mathrm{v}}$ and $\mathrm{F}_{\mathrm{v}}$ be as defined in (4.5),

let $\quad \mathrm{P}_{\mathrm{v}}=\square\left(\mathrm{vp}_{\mathrm{v}}\right)$

then, necessary conditions for the implication (4.10) to be satisfied are,

$$
\lambda_{\mathrm{v}}=\square\left(\frac{\mathrm{v}}{\left(1+\mathrm{F}_{\mathrm{v}}\right)}\right), \quad \lambda_{\mathrm{v}}=\square\left(\frac{\mathrm{v}^{(1+\delta-1 / \mathrm{k})}}{\mathrm{f}_{\mathrm{v}} \mathrm{p}_{\mathrm{v}}}\right), \quad \Delta \lambda_{\mathrm{v}}=\square\left(\frac{\mathrm{v}^{(1+\delta-1 / \mathrm{k})}}{1+\mathrm{F}_{\mathrm{v}+1}}\right)
$$

Proof: The proof follows from Theorem 2 by putting

$\mathrm{q}_{\mathrm{n}}=\mathrm{r}_{\mathrm{n}}=1$, for all $\mathrm{n}$, noticing that (2.3) is satisfied as in the case of (4.17).

Cor. 10

By putting $\delta=0$, corollary. 9 reduces to Cor. 2.5 of SULAIMAN[3].

\section{Cor. 11}

Let $\mathrm{f}_{\mathrm{v}}$ and $\mathrm{F}_{\mathrm{v}}$ be as defined in (4.1), Let (2.3), (2.13) and (4.2) be all satisfied, then, necessary conditions for the implication (4.18) to be satisfied are,

$$
\lambda_{v}=\square\left(\frac{Q_{v} Q_{v-1} r_{v}}{\left(1+F_{v}\right) R_{v} q_{v}}\right), \quad \lambda_{v}=\square\left(\frac{v^{(1+\delta)-1 / k} r_{v} Q_{v}}{f_{v} R_{v}}\right), \quad \Delta \lambda_{v}=\square\left(\frac{v^{(1+\delta)-1 / k} r_{v} Q_{v}}{\left(1+F_{v+1}\right) R_{v}}\right)
$$

Proof: The proof follows from Theorem 2, by putting $\mathrm{p}_{\mathrm{n}}=1 \forall \mathrm{n}$.

Cor. 12

When $\delta=0$, corollary. 11 reduces to Cor. 2.6 of SULAIMAN[3].

Acknowledgement: I am very thankful to Dr. Rajiv Sinha (Associate Professor, S.M.P.G. College ,Chandausi ,U.P., India ), whose great inspiration lead me to complete this paper.

\section{References}

[1] DAS, G.-Tauberian theorem for absolute Nörlund summability, 'Procidings of the London Math. Soc. Vol. 19, No. 2, pp 357-384, 1969'.

[2] FLETT, T.M. - On an extension of absolute summability andsome theorems of Littlewood and Paley, Prociding of London Math. Soc. Vol. 7 , No.1, pp.-113-141, 1957.

[3] SULAIMAN, W.T. - Note on product summability of infinite series, International J. of Math. And Math. Sci. Vol. ,2008, Article I.D. 372604 . 